\title{
Analysis of Metallo- $\beta$-lactamases, oprD Mutation, and Multidrug Resistance of $\beta$-lactam Antibiotic-Resistant Strains of Pseudomonas aeruginosa Isolated from Southern China
}

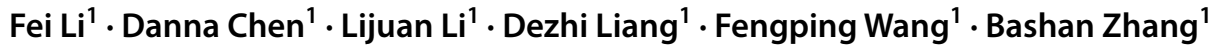

Received: 18 November 2019 / Accepted: 25 July 2020 / Published online: 12 August 2020

(c) The Author(s) 2020

\begin{abstract}
The purpose of this study was to analyze the metallo- $\beta$-lactamases (MBLs) genotype and oprD mutations of the $\beta$-lactam antibiotic-resistant Pseudomonas aeruginosa (PA) strains isolated from southern China. We collected 110 strains of $\beta$-lactam antibiotic-resistant PA from 2 hospitals during January 2016-December 2017 from Dongguan, South China. MBLs were detected, amplified, and typed using EDTA disc synergy test, PCR, and Sanger gene sequencing. The mutations and expression levels of $\operatorname{opr} D$ were detected using Sanger gene sequencing and qPCR. A total of 16.36\% (18/110) $\beta$-lactam antibiotic-resistant PA strains produced MBLs, and the main genotypes of MBLs were IMP-25, VIM-2, and SIM-2. Sanger gene sequencing results showed that 107 of the 110 strains harbored mutations in $\operatorname{opr} D$ sequence, while 3 strains were negative for $\operatorname{opr} D$ amplification (2.73\%). Among the 107 strains with positive amplification (97.27\%), the rate of intentional mutations (including deletions, insertions, and premature stop codons) was $93.46 \%$ (100/107) and that of no disrupted mutation was 6.54\% (7/107). qPCR analysis confirmed that the expression level of the OprD protein in the 7 strains of no disrupted mutation was significantly reduced. Among the $\beta$-lactam antibiotic-resistant PA strains in southern China, $16.36 \%$ were positive for MBLs. The loss rate of $\operatorname{oprD}$ was $2.73 \%$, and almost all PA strains showed $\operatorname{oprD}$ amplification variation or transcription downregulation. Thus, impaired $\operatorname{or} D$ expression and MBLs production may be some of the mechanisms of $\beta$-lactam antibiotic-resistance of PA strains in southern China.
\end{abstract}

\section{Introduction}

Pseudomonas aeruginosa (PA) is a common opportunistic pathogen that can cause infections in immunocompromised patients or in patients with burns. Since the 1990s, the incidence of clinical isolates of PA has increased [1]. Clinically, $\beta$-lactam antibiotics are one of the most commonly used antibiotics for treatment of PA infections. However, the use of antibiotics for a long time may increase the drug

Fei Li and Danna Chen have contributed equally to this work.

Electronic supplementary material The online version of this article (https://doi.org/10.1007/s00284-020-02148-3) contains supplementary material, which is available to authorized users.

Bashan Zhang

zbsdgry@163.com

1 Clinical Laboratory, Affiliated Dongguan People's Hospital, Southern Medical University, No.3 Xinguchong Wandao South Road, Wangjiang District, Dongguan 523059, Guangdong, China resistance rate and multidrug resistance to $\beta$-lactam antibiotics in PA, thereby leading to difficulties in the treatment of PA infections [2,3]. China's monitoring report suggests that PA resistance to various antimicrobial agents is quite high in 2016 [4]. Therefore, the study of $\beta$-lactam antibiotic resistance and multidrug resistance mechanisms in PA is conducive to the prevention and treatment of PA infection.

Metallo- $\beta$-lactamases (MBLs), also known as class B $\beta$-lactamases, are carbapenemases that are usually identified in PA. MBLs can effectively hydrolyze $\beta$-lactams, including carbapenems, and aminoglycosides, and other antibiotics such as quinolones [5]. Various types of MBLs have been identified in PA, including IMP, VIM, SPM-1, GIM-1, SIM-1, NDM-1, and FIM-1. The production of MBLs is considered to be one of the important mechanisms for PA resistance $[6,7]$. In addition, outer membrane porins, like OprD, that uptake amino acids, peptides, and carbapenems undergo insertion, frameshift, and mutation-mediated gene inactivation or expression suppression, which can increase carbapenem resistance in PA [8-11]. 
Although the production of MBLs and the inactivation of $\operatorname{opr} D$ in PA resistant to carbapenems have been reported in specific countries and regions such as Korea or in specific populations such as patients with fibrosis in South Korea [1,9], the drug resistance mechanisms of $\beta$-lactam antibiotic-resistant strains of PA in China have not been still elucidated. The aim of this study was to analyze the drug susceptibility profiles of 110 isolates of $\beta$-lactam antibiotic-resistant PA from Dongguan, South China, and to determine the expression of MBLs and $o p r D$ for revealing the drug resistance mechanisms in $\beta$-lactam antibiotic-resistant PA. This study is expected to provide valuable information on the drug resistance in PA for the diagnosis and treatment of infections caused by PA.

\section{Materials and Methods}

\section{Bacterial Isolates and Susceptibility Testing}

Pseudomonas aeruginosa samples isolated from the patients who visited Dongguan People's Hospital and Dongguan Donghua Hospital from 2016 to 2017 were stored in skimmed milk at $-70{ }^{\circ} \mathrm{C}$. The strains were reconstituted from skimmed milk to a blood plate, and a single colony was picked from the blood plate. Antibiotic susceptibility and drug resistance of each strain was measured using the VITEK 2 Compact system (BioMérieux, Lyon, France). Drugs that were used for antibiotic susceptibility determination were ampicillin, ampicillin/sulbactam, piperacillin/tazobactam, cefazolin, cefotetan, ceftazidime, ceftriaxone, cefepime, imipenem, amikacin, gentamicin, tobramycin, ciprofloxacin, levofloxacin, nitrofurantoin, meropenem, and compound sulfamethoxazole. Multiple $\beta$-lactam antibiotic-resistant samples were selected for subsequent analyses.

\section{Pulsed-Field Gel Electrophoresis (PFGE)}

A single colony was picked, inoculated on a nutrient plate, and incubated overnight at $37^{\circ} \mathrm{C}$. The concentration of the bacterial suspension was adjusted with a DENSIMAT turbidimeter with a turbidity of 3.8-4.2. Next, $1 \%$ Seakem Gold Glue was prepared. Bacterial genomic DNA was isolated on the gel block. The enzyme was digested with $40 \mathrm{U}$ SpeI (Takara Bio, Otsu, Japan) endonuclease and incubated at $37^{\circ} \mathrm{C}$ for $4 \mathrm{~h}$. PFGE was performed in a CHEF-DRIII (BioRad Laboratories, CA, USA) electrophoresis apparatus. The electrophoresis parameters were 5-15 s for $9 \mathrm{~h}$ and $15-50 \mathrm{~s}$ for $9 \mathrm{~h}$. After the electrophoresis was completed, the gel was stained with a GelRed nucleic acid dye. Correlation analysis of PFGE patterns was performed using the BioNumerics software version 4.0 (Applied Maths, Sint-Martens-Latem, Belgium).

\section{Ethylenediaminetetraacetic Acid (EDTA) Disc Synergy Test}

Since divalent cations (usually zinc) act as cofactors for maintaining the MBL enzyme activity and are inhibited by EDTA, EDTA is often used to detect MBL activity in PA [12]. Each isolate was inoculated in the Mueller-Hinton broth at $1 \times 10^{6} \mathrm{CFU} / \mathrm{mL}$ and then inoculated on the agar medium with a cotton swab. Two discs (antibiotic disc and antibiotic + EDTA disc) were placed on the agar surface using sterile forceps with a spacing of approximately $30 \mathrm{~mm}$. The EDTA disc was prepared by adding $250 \mu \mathrm{L}$ of neutral EDTA to imipenem. The bacteriostatic inhibition zone was observed by incubation at $37{ }^{\circ} \mathrm{C}$ for $24 \mathrm{~h}$. When the inhibition zone was $>4 \mathrm{~mm}$ in diameter, the strain was considered to be an MBL-positive phenotype. In addition, a control tray containing only EDTA was used to determine the activity of EDTA to ensure that the test isolate was inhibited by EDTA without causing false-positive results.

\section{Mbls and oprD Gene Amplification}

DNA of each bacterial isolate was extracted using the TaKaRa Ex Taq® Hot Start Version (RR006Q, TaKaRa, Japan). Fragments of $m b l s$ and full-length oprD were amplified with the primers presented in Supplementary Table 1. The PCR program was set as follows: initial denaturation at $94{ }^{\circ} \mathrm{C}$ for $5 \mathrm{~min}$; followed by 30 cycles of $94{ }^{\circ} \mathrm{C}$ for $30 \mathrm{~s}, 52^{\circ} \mathrm{C}$ for $40 \mathrm{~s}$, and $72{ }^{\circ} \mathrm{C}$ for $50 \mathrm{~s}$; and final extension at $72{ }^{\circ} \mathrm{C}$ for $5 \mathrm{~min}$. The PCR products were sequenced for gene typing using Sanger sequencing (Sangon Biotech, Shanghai, China).

\section{OrpD Mutation and Expression}

Total RNA was isolated from the $\beta$-lactam antibioticresistant strains using the Trizol reagent (Thermo Fisher Scientific, IL, USA) according to the standard protocol. cDNA was synthesized using M-MLV Reverse Transcriptase (Promega, WI, USA). OprD was amplified using the Bestar qPCR RT Kit (DBI Bioscience, Ludwigshafen, Germany) according to the manufacturer's instructions. The relative expression of $\operatorname{opr} D$ was normalized to that of the PAO1 standard strain and calculated by the $2^{-\Delta \Delta \mathrm{ct}}$ method. RpsL was used as the internal control. 


\section{Results}

\section{Drug Sensitivity and Resistance of $\beta$-Lactam Antibiotic- Resistant PA}

The 110 strains of PA isolated from Dongguan were resistant to $\beta$-lactams, fluoroquinolones, tetracyclines, and sulfonamides. Among them, 100\%, 100\%, 98.18\%, 97.27\%, and $96.36 \%$ of the isolates were resistant to imipenem, ampicillin, nitrofurantoin, compound sulfamethoxazole, and cefazolin, respectively. Approximately, 31.82\%, $27.27 \%$, and $26.36 \%$ of the strains were resistant to ceftazidime, ciprofloxacin, and levofloxacin, respectively. The resistance rate to amikacin was $10.00 \%$, and the resistance rates to tobramycin, gentamicin, cefepime, and piperacillin/tazobactam were $17.27 \%, 19.09 \%, 21.82 \%$, and $21.82 \%$, respectively (Table 1 ).

\section{MBLs Production in $\beta$-Lactam Antibiotic-Resistant PA}

The EDTA disc synergy test showed that there were 18 (16\%) isolates that were MBL-positive PA. Further typing results showed that 9 isolates were IMP-25 (8.18\%), 8 isolates were VIM-2 (7.27\%), and 4 isolates were SIM-2 (3.64\%); in addition, in 3 isolates, both VIM-2 and SIM-2 were identified. The sequence alignment of these isolates in the GenBank was found to be identical

Table 1 Sensitivity of $\beta$-lactam antibiotic-resistant Pseudomonas aeruginosa (PA) to commonly used antibiotics

\begin{tabular}{lll}
\hline Antibiotics & Sensitivity & Resistance \\
\hline Imipenem & $0(0 \%)$ & $110(100 \%)$ \\
Ampicillin & $0(0)$ & $110(100 \%)$ \\
Nitrofurantoin & $2(1.82 \%)$ & $108(98.18 \%)$ \\
Compound sulfamethoxazole & $3(2.73 \%)$ & $107(97.27 \%)$ \\
Cefazolin & $0(0 \%)$ & $106(96.36 \%)$ \\
Cefotetan & $2(1.81 \%)$ & $104(94.55 \%)$ \\
Ampicillin/sulbactam & $7(6.36 \%)$ & $103(93.64 \%)$ \\
Ceftriaxone & $6(5.45 \%)$ & $100(90.91 \%)$ \\
Meropenem & $14(12.73 \%)$ & $82(74.54 \%)$ \\
Ceftazidime & $54(49.09 \%)$ & $35(31.82 \%)$ \\
Ciprofloxacin & $64(58.19 \%)$ & $30(27.27 \%)$ \\
Levofloxacin & $69(62.73 \%)$ & $29(26.36 \%)$ \\
Piperacillin/tazobactam & $47(42.73 \%)$ & $24(21.82 \%)$ \\
Cefepime & $75(68.18 \%)$ & $24(21.82 \%)$ \\
Gentamicin & $78(70.91 \%)$ & $21(19.09 \%)$ \\
Tobramycin & $83(75.45 \%)$ & $19(17.27 \%)$ \\
Amikacin & $96(87.27 \%)$ & $11(10 \%)$ \\
\hline
\end{tabular}

to IMP-25 (EU352796), VIM-2 (AF191564), and SIM-2 (KT013203).

\section{OprD Variation, Phylogenetic Analysis, and Expression Analysis of $\beta$-Lactam Antibiotic-Resistant PA}

The phylogenetic analysis of the $110 \beta$-lactam antibioticresistant PA isolates according to the oprD sequence revealed that the gene could be divided into 4 classes, and each class had a sequence with high homology in GenBank. The standard strain (PAO1) reference sequence was in the third class (Fig. 1).

The oprD typing results showed that 107 isolates were positive for $\operatorname{opr} D$, while 3 were negative. The rate of $\operatorname{opr} D$ deletion rate was $2.73 \%$. Further sequencing of the oprDpositive isolates revealed that there were no disrupted mutations in 7 isolates, while there were frameshift mutations in 84 isolates and single-base single-point mutations in 16 isolates with premature stop codons (Table 2).

The mRNA expression levels of $\operatorname{opr} D$ were detected in the 7 non-disruptive mutant isolates (numbered: 5, 11, 18, $23,43,81$, and 107). The qPCR results showed a significant decrease in the expression of $\operatorname{opr} D$ in all the isolates compared to that in the carbapenem-susceptible PAO1 strains (Fig. 2). In addition, we detected the expression of oprD in 10 imipenem-sensitive PA strains, however, did not find any strains with reduced oprD expression (data not shown).

\section{Discussion}

There are only few studies on the drug resistance mechanisms of $\beta$-lactam antibiotic-resistant PA in southern China. One study had shown a mutation in oprD in imipenem-resistant PA in southern China [9]. Compared to the isolates in the previous study, the isolates in this study were characterized by multiple $\beta$-lactam antibiotic-resistance, and we analyzed the production and typing of MBLs from these isolates. We found that 18 of the 110 isolates of the $\beta$-lactam antibioticresistant PA in Dongguan, China, produced MBL, and 107 isolates were positive for $o p r D$ amplification. Further phylogenetic analysis of $\beta$-lactam antibiotic-resistant PA based on the $\operatorname{opr} D$ sequence revealed that these resistant isolates were mainly divided into 4 groups. $O p r D$ sequence analysis revealed a variation in $\operatorname{opr} D$ sequence of most $\beta$-lactam antibiotic-resistant PA strains. Among them, 84 isolates showed frameshift mutations, 16 showed premature stop codons, and 7 showed non-disruptive mutations. In particular, the 7 nondisruptive mutants showed a significant decrease in the gene expression level of $o p r D$.

Currently, acquired MBLs include IMP, VIM, SPM, GIM, SIM, AIM, KHM, DIM, NDM, with IMP and VIM 
Fig. 1 Pulsed-field gel electrophoresis (PFGE) profiles showing $\beta$-lactam antibiotic-resistant Pseudomonas aeruginosa (PA) isolates

being the most common ones [13]. IMP, VIM, SPM, SIM, GIM, and NDM are of epidemiological and clinical significance in antibiotic resistance [14-16]. A previous national PA multidrug resistance test across 27 hospitals-mostly from southeastern China and southern China-shows that $8.2 \%$ of the strains produce MBLs, with IMP-9 being the most common one, followed by VIM-2 [17]. Another study shows that approximately $13(35.1 \%)$ of the 37 multidrugresistant PA strains isolated from the Hunan Province, China, produced IMP-4 and VIM-2 [18]. In our study, 18 resistant isolates $(16.36 \%)$ carried MBLs-mainly IMP25, VIM-2, and SIM-2. This result suggested that IMP25 , VIM-2, and SIM-2 played important roles in $\beta$-lactam antibiotic-resistance. In terms of the major epidemic types, our research suggested that the dominant type of MBLs in the southern region differed from the dominant types in the Hunan Province and the rest of the country. Simultaneously, there was also a consensus that the IMP type was dominant, followed by the VIM-2 type. This result was consistent with worldwide trends, indicating a potential association of IMP and VIM with PA multidrug resistance.

The inactivation of $\operatorname{oprD}$ is considered to be one of the mechanisms of PA resistance. Both sequence insertion and mutation can affect the activity of $\operatorname{opr} D[10,19]$. Our study found that $\operatorname{opr} D$ sequence of 107 isolates was mutated, while 3 isolates showed gene loss. In particular, the PCR results verified that the expression of $\operatorname{orr} D$ was decreased in the isolates with non-disruptive mutations, suggesting that the function of the $\operatorname{or} D$ of these isolates was impaired. In addition, 3 isolates showed negative $\operatorname{opr} D$ amplification, suggesting the absence of $\operatorname{opr} D$. Thus, we hypothesized that $\operatorname{opr} D$, including positive or negative $\operatorname{opr} D$ amplification, might show abnormality in expression and function. The expression of $\operatorname{opr} D$ has been reported to be downregulated in multidrug-resistant PA in China [9, 18]. Our study further provided evidence that oprD expression was reduced in the non-disruptive mutant isolates. Studies have suggested that more than $50 \%$ of isolates have consistent characteristics of MBL and oprD [20]. Our study also showed a similar trend of both impaired MBLs production and mutated $\operatorname{oprD}$ sequence, which further supported this opinion.

\section{Conclusion}

In summary, we analyzed the MBLs production of the $\beta$-lactam antibiotic-resistant PA strains in southern China and the variations in the $\operatorname{opr} D$ sequence, which were the 2 important mechanisms of PA resistance. Our results indicated that the drug resistance mechanism of

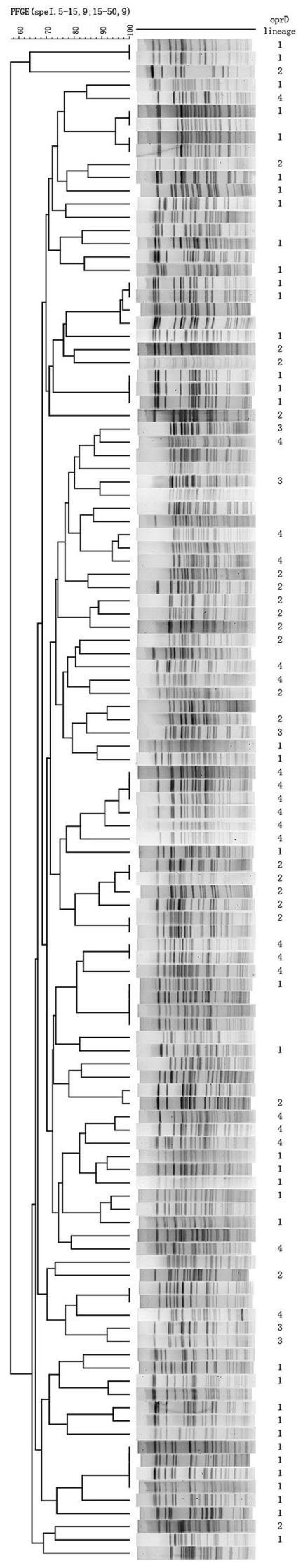


Table 2 Mutation of $\operatorname{opr} D$ in $\beta$-lactam antibiotic-resistant Pseudomonas aeruginosa (PA)

\begin{tabular}{llcl}
\hline Type of mutation & & Number of isolates & Percentage (\%) \\
\hline Frameshift & 1-bp insertion & 7 & 6.36 \\
& More than 1-bp insertion & 5 & 4.55 \\
& 1-bp deletion & 8 & 7.27 \\
& More than 1-bp deletion & 56 & 50.91 \\
& Insertion & 8 & 7.27 \\
Premature stop & Single mutation & 16 & 14.55 \\
Negative by PCR & Unknown large change & 3 & 2.73 \\
No disrupted mutation & Amino acid changes & 7 & 6.36 \\
Total & & 110 & 100
\end{tabular}

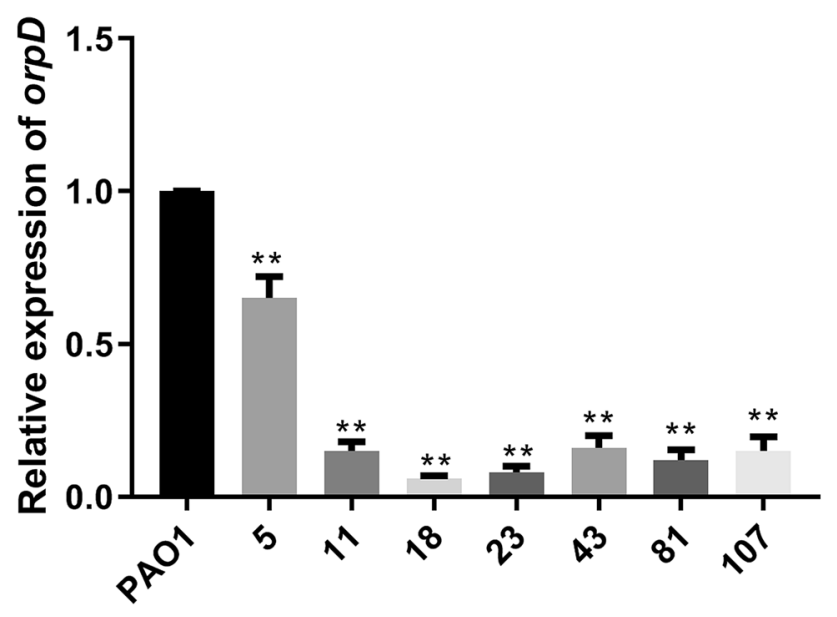

Fig. 2 Relative expression of oprD expression in $\beta$-lactam-resistant Pseudomonas aeruginosa (PA) with no disruptive mutation

$\beta$-lactam antibiotic-resistant PA in southern China might be attributed to the impaired oprD expression and MBLs production. Our study further provided evidence of the MBLs production and the variation in the oprD sequence as the clue for the treatment of infections caused by $\beta$-lactam multidrug-resistant PA in southern China.

Acknowledgements The study was supported by the Dongguan Social Science and Technology Development Project (No.2016108101042).

Author Contributions The study concept and design, and the manuscript revision were performed by BZ. The experiments performance, data analysis and the manuscript draft were performed by FL and DC. The study design, study implementation and manuscript revision were performed by LL, DL and FW. All authors read and approved the final manuscript.

Data Availability The datasets generated during and/or analyzed during the current study are available from the corresponding author on reasonable request.

\section{Compliance with Ethical Standards}

Conflict of interest The authors declare that they have no conflict of interest.

Research Involving Human Participants and/or Animals This article does not contain any studies with human participants or animals performed by any of the authors.

Open Access This article is licensed under a Creative Commons Attribution 4.0 International License, which permits use, sharing, adaptation, distribution and reproduction in any medium or format, as long as you give appropriate credit to the original author(s) and the source, provide a link to the Creative Commons licence, and indicate if changes were made. The images or other third party material in this article are included in the article's Creative Commons licence, unless indicated otherwise in a credit line to the material. If material is not included in the article's Creative Commons licence and your intended use is not permitted by statutory regulation or exceeds the permitted use, you will need to obtain permission directly from the copyright holder. To view a copy of this licence, visit http://creativecommons.org/licenses/by/4.0/.

\section{References}

1. Tomas M, Doumith M, Warner M, Turton JF, Beceiro A, Bou G, Livermore DM, Woodford N (2010) Efflux pumps, OprD porin, AmpC beta-lactamase, and multiresistance in Pseudomonas aeruginosa isolates from cystic fibrosis patients. Antimicrob Agents Chemother 54(5):2219-2224. https://doi.org/10.1128/aac.00816 $-09$

2. Palavutitotai N, Jitmuang A, Tongsai S, Kiratisin P, Angkasekwinai N (2018) Epidemiology and risk factors of extensively drug-resistant Pseudomonas aeruginosa infections. PLoS ONE 13(2):e0193431. https://doi.org/10.1371/journal.pone.0193431

3. Siriyong T, Murray RM, Bidgood LE, Young SA, Wright F, Parcell BJ, Voravuthikunchai SP, Coote PJ (2019) Dual beta-lactam combination therapy for multi-drug resistant Pseudomonas aeruginosa infection: enhanced efficacy in vivo and comparison with monotherapies of penicillin-binding protein inhibition. Sci Rep 9(1):9098. https://doi.org/10.1038/s41598-019-45550-Z

4. Fupin H, Guo Y, Zhu D, Wang F, Jiang X, Yingchun X, Zhang X, Zhang Z, Ping J (2017) CHINET surveillance of bacterial resistance across China: report of the results in 2016. Chin J Infect Chemother 17(5):481-491 
5. Palzkill T (2013) Metallo-beta-lactamase structure and function. Ann N Y Acad Sci 1277:91-104. https://doi.org/10.111 1/j.1749-6632.2012.06796.x

6. Lohans CT, Freeman EI, Groesen EV, Tooke CL, Hinchliffe P, Spencer J, Brem J, Schofield CJ (2019) Mechanistic insights into beta-lactamase-catalysed carbapenem degradation through product characterisation. Sci Rep 9(1):13608. https://doi.org/10.1038/ s41598-019-49264-0

7. Tabasi M, Azizian R, Eskandarion MR, Habibi M, Asadi Karam MR (2017) Detection of Metallo- $\beta$-lactamases (MBLs) producing Pseudomonas aeruginosa isolates in Tehran hospitals. Iran J Med Microbiol Infect Dis 5(3):47-50

8. Li H, Luo YF, Williams BJ, Blackwell TS, Xie CM (2012) Structure and function of OprD protein in Pseudomonas aeruginosa: from antibiotic resistance to novel therapies. Int J Med Microbiol 302(2):63-68. https://doi.org/10.1016/j.ijmm.2011.10.001

9. Fang ZL, Zhang LY, Huang YM, Qing Y, Cao KY, Tian GB, Huang X (2014) OprD mutations and inactivation in imipenem-resistant Pseudomonas aeruginosa isolates from China. Infect Genet Evol 21:124-128. https://doi.org/10.1016/j.meegi d.2013.10.027

10. Kim CH, Kang HY, Kim BR, Jeon H, Lee YC, Lee SH, Lee JC (2016) Mutational inactivation of OprD in carbapenem-resistant Pseudomonas aeruginosa isolates from Korean hospitals. J Microbiol 54(1):44-49. https://doi.org/10.1007/s12275-016-5562-5

11. Sanbongi Y, Shimizu A, Suzuki T, Nagaso H, Ida T, Maebashi K, Gotoh N (2009) Classification of oprD sequence and correlation with antimicrobial activity of carbapenem agents in Pseudomonas aeruginosa clinical isolates collected in Japan. Microbiol Immunol 53(7):361-367. https://doi.org/10.111 1/j.1348-0421.2009.00137.x

12. Maltezou HC (2009) Metallo-beta-lactamases in Gram-negative bacteria: introducing the era of pan-resistance? Int J Antimicrob Agents 33(5):405.e1-7. https://doi.org/10.1016/j.ijantimica g.2008.09.003

13. Belles A, Bueno J, Rojo-Bezares B, Torres C, Javier Castillo F, Saenz Y, Seral C (2018) Characterisation of VIM-2-producing Pseudomonas aeruginosa isolates from lower tract respiratory infections in a Spanish hospital. Eur J Clin Microbiol Infect Dis 37(10):1847-1856. https://doi.org/10.1007/s10096-018-3318-3

14. Cornaglia G, Giamarellou H, Rossolini GM (2011) Metallo-betalactamases: a last frontier for beta-lactams? Lancet Infect Dis 11(5):381-393. https://doi.org/10.1016/s1473-3099(11)70056-1

15. Hong DJ, Bae IK, Jang IH, Jeong SH, Kang HK, Lee K (2015) Epidemiology and characteristics of metallo-beta-lactamase-producing Pseudomonas aeruginosa. Infect Chemother 47(2):81-97. https://doi.org/10.3947/ic.2015.47.2.81

16. Ghasemian A, Salimian Rizi K, Rajabi Vardanjani H, Nojoomi F (2018) Prevalence of clinically isolated metallo-beta-lactamaseproducing Pseudomonas aeruginosa, coding genes, and possible risk factors in Iran. Iran J Pathol 13(1):1-9

17. Fan X, Wu Y, Xiao M, Xu ZP, Kudinha T, Bazaj A, Kong F, $\mathrm{Xu}$ YC (2016) Diverse genetic background of multidrug-resistant Pseudomonas aeruginosa from Mainland China, and emergence of an extensively drug-resistant ST292 clone in Kunming. Sci Rep 6:26522. https://doi.org/10.1038/srep26522

18. Li J, Zou M, Dou Q, Hu Y, Wang H, Yan Q, Liu WE (2016) Characterization of clinical extensively drug-resistant Pseudomonas aeruginosa in the Hunan province of China. Ann Clin Microbiol Antimicrob 15(1):35. https://doi.org/10.1186/s12941-016-0148-y

19. Sun $\mathrm{Q}, \mathrm{Ba} Z$, Wu G, Wang $\mathrm{W}$, Lin $\mathrm{S}$, Yang H (2016) Insertion sequence ISRP10 inactivation of the oprD gene in imipenemresistant Pseudomonas aeruginosa clinical isolates. Int J Antimicrob Agents 47(5):375-379. https://doi.org/10.1016/j.ijantimica g.2016.02.008

20. Seqqat R, Vallejo MJ, Grijalva M, Calvopiña K (2017) AmpC, oprD expression analysis in $\beta$-lactam resistant Pseudomonas aeruginosa clinical isolates 1 from a tertiary level hospital in Ecuador. Rev Ecuat de Med y Ciencias Biol 38(1):35-43. https://doi. org/10.26807/remcb.v38i1.19

Publisher's Note Springer Nature remains neutral with regard to jurisdictional claims in published maps and institutional affiliations. 